\title{
Erratum to: A prospective cohort study of depression course, functional disability, and NEET status in help-seeking young adults
}

\author{
Bridianne O'Dea ${ }^{1,2} \cdot$ Rico S. C. Lee $^{1} \cdot$ Patrick D. McGorry ${ }^{3,4} \cdot$ Ian B. Hickie $^{1}$. \\ Jan $\operatorname{Scott}^{5} \cdot$ Daniel F. Hermens ${ }^{1} \cdot$ Arnstein Mykletun $^{6,9,10} \cdot$ Rosemary Purcell $^{4,7}$. \\ Eoin Killackey $^{3,4} \cdot$ Christos Pantelis $^{8}$ - G. Paul Amminger ${ }^{4}$ Nicholas Glozier ${ }^{1}$
}

Published online: 7 February 2017

C) Springer-Verlag Berlin Heidelberg 2017

\section{Erratum to: Soc Psychiatry Psychiatr \\ Epidemiol (2016) 51:1395-1404 \\ DOI 10.1007/s00127-016-1272-x}

In the original publication of the article, coauthor name has been published incorrectly. The correct co-author name should be "Arnstein Mykletun".

The online version of the original article can be found under doi:10.1007/s00127-016-1272-x.

\section{Bridianne O'Dea}

b.odea@blackdog.org.au

1 Brain and Mind Centre, University of Sydney, Sydney, NSW, Australia

2 Black Dog Institute, Faculty of Medicine, University of New South Wales, Sydney, NSW, Australia

3 Orygen, The National Centre of Excellence in Youth Mental Health, Melbourne, VIC, Australia

4 Centre for Youth Mental Health, University of Melbourne, Melbourne, VIC, Australia

5 Institute of Neuroscience, Newcastle University, Newcastle-upon-Tyne, UK

6 Department of Mental Health and Suicide, Norwegian Institute of Public Health, Oslo, Norway
7 Centre for Forensic Behavioural Science, Faculty of Health, Arts and Design, Swinburne University of Technology, Melbourne, VIC, Australia

8 Melbourne Neuropsychiatry Centre, Department of Psychiatry, University of Melbourne and Melbourne Health, Melbourne, VIC, Australia

9 Department of Community Medicine, University of Troms $\varnothing$, Tromso, Norway

10 Center for Work and Mental Health, Nordland Hospital Trust, Bodo, Norway 